\title{
Novel origin of cerebral phaeohyphomycosis: A case report
}

\author{
Christopher Daniele MD, Miriam Ferguson MD, Cooper Phillips MD, Jinesh Lachmansingh MD, \\ Jacob Nichols MD, John Fisher, Brady Holstead MS, Akwasi Opoku BA
}

\begin{abstract}
Cerebral phaeohyphomycosis is infection of the central nervous system (CNS) caused by dematiaceous fungi. The most common mechanisms of infection are by direct extension from the paranasal sinuses or by hematogenous spread. The mortality rate is high and ranges from $50 \%-79 \%$ in the limited studies on this infection. Our case describes a young man without any preexisting disease who acquired cerebral phaeohyphomycosis following nasal inhalation of garden mushrooms. Despite aggressive treatment, he ultimately died due to this severe infection. Our case report discusses our treatment approach and reviews the literature on this deadly infection.
\end{abstract}

Keywords: fungal infection, cerebral abscess, Bipolaris, phaeohyphomycosis

\section{INTRODUCTION}

Phaeohyphomycosis refers to an infection caused by phaeoid (also called dematiaceous or pigmented) fungi; the clinical presentation of which can be extremely diverse. Case reports and literature reviews have shown that patients with phaeohyphomycosis can present with a wide variety of infections, such as sinusitis, endocarditis, endophthalmitis, keratitis, endarteritis, osteomyelitis, meningoencephalitis, peritonitis, otitis media, and fungemia. These infections are often seen in the immunocompromised, but many case reports have been published involving seemingly immunocompetent patients. Our case involves a young, healthy man who presented for evaluation after reported inhalation of garden mushrooms intranasally that resulted in cerebral phaeohyphomycosis.

\section{CASE}

The patient is an 18-year-old man with no past medical or surgical history who presented to an

Corresponding author: Christopher Daniele Contact Information: Christopher.daniele@ttuhsc.edu DOI: 10.12746/swrccc.v8i33.637 outside hospital facility complaining of two weeks of headache and one day of nausea, vomiting, and "not feeling right." He underwent a computed tomography (CT) scan of the head and neck at the outside hospital facility which demonstrated a large right frontal mass. He was subsequently transferred to University Medical Center in Lubbock, TX, for neurosurgical evaluation.

The patient was admitted to the hospitalist medicine service and was evaluated by our neurosurgical service. Magnetic resonance imaging (MRI) of the brain demonstrated an "expansile mass in the right frontal lobe with hemorrhagic and necrotic changes [with] findings worrisome for high-grade glial neoplasm;" he also underwent a repeat head CT for clinical deterioration after the MRI that demonstrated new findings of subfalcine herniation. He was subsequently taken to the operating room emergently by the neurosurgery service for a right frontal craniotomy and lobectomy. Intraoperatively, his cerebrospinal fluid was described as "murky" with "significant proteinaceous material" and "an early preliminary diagnosis consistent with a fungal abscess." Intraoperative specimens were also sent for bacterial and fungal cultures. He was empirically started on broad antifungal coverage with amphotericin $B$ and isavuconazonium sulfate based on infectious disease service recommendations. 


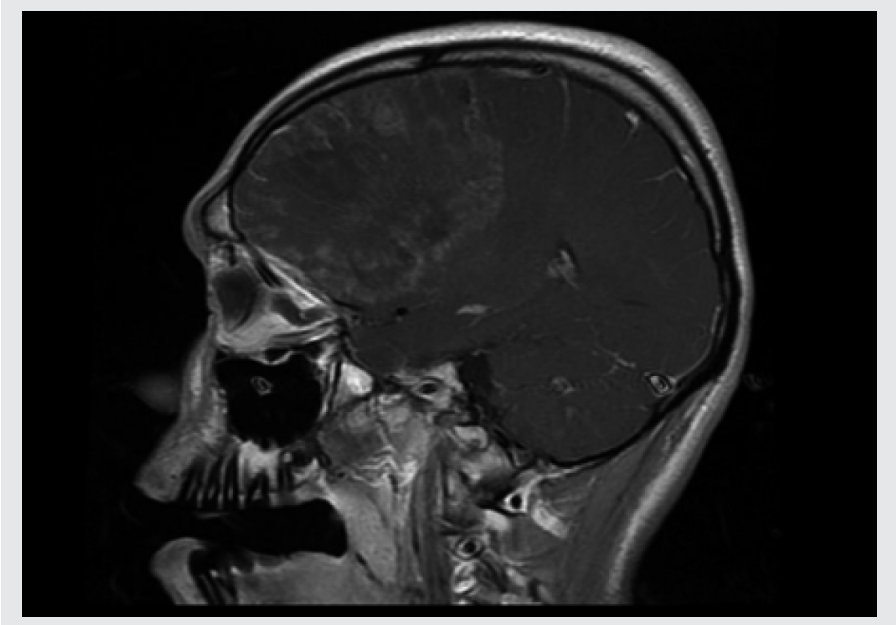

Figure 1. Sagittal MRI image showing large frontal mass.

Following surgery, he was taken to the medical intensive care unit for post-operative management. His immediate post-operative course was complicated by new onset seizures with new imaging showing vasogenic edema and a $6 \mathrm{~mm}$ right-to-left shift. The patient was then taken back to the operating room for bilateral external ventricular drain (EVD) placement.

After ventricular drain placement, he was transferred to our neuro-anesthesia intensive care unit for

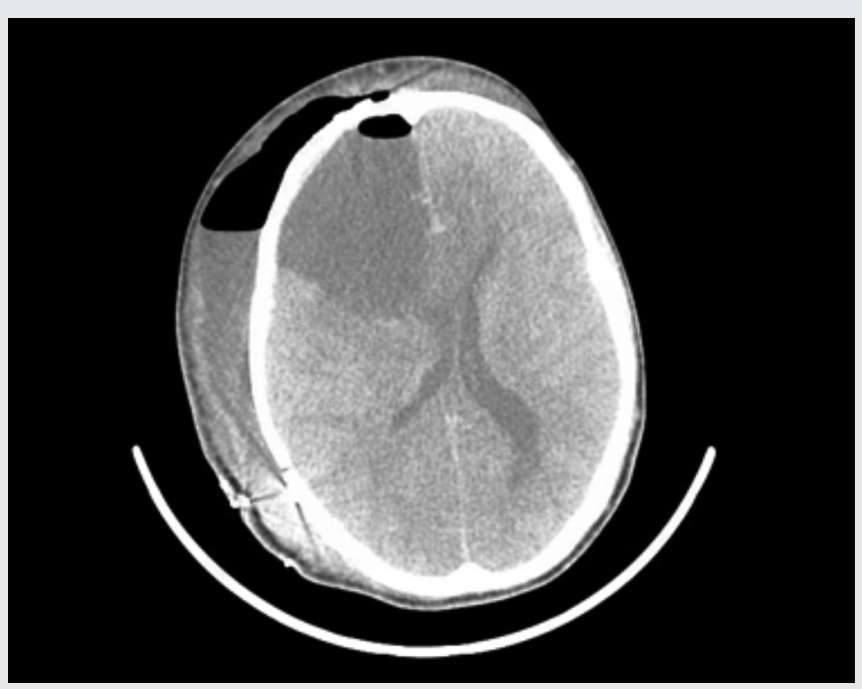

Figure 2. Axial MRI image showing large frontal mass.

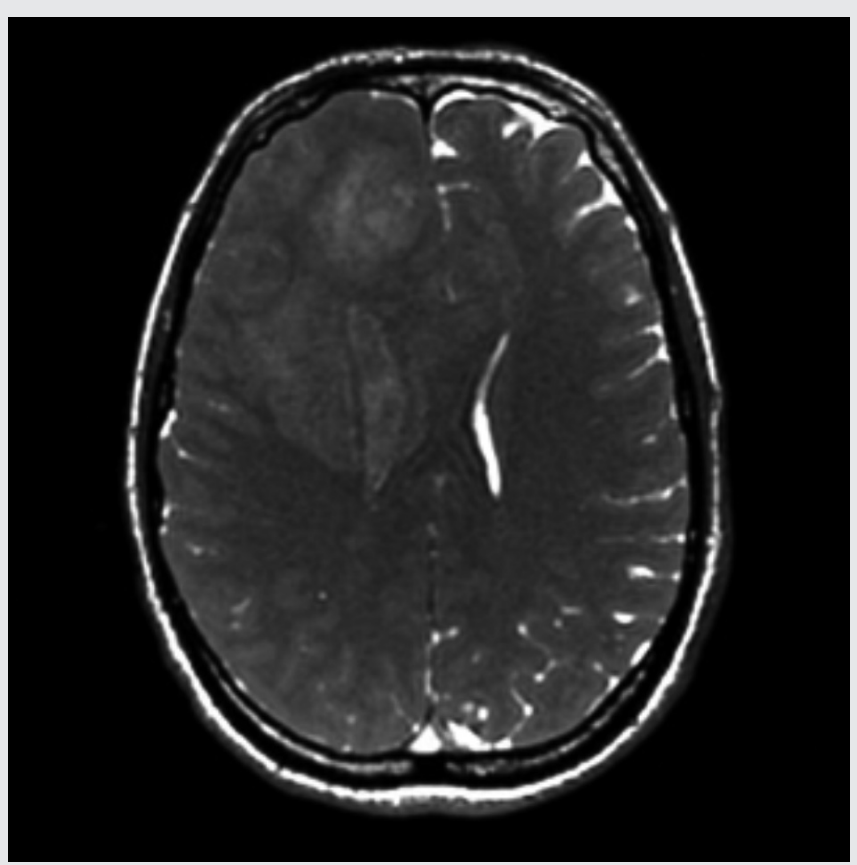

Figure 3. Axial CT Scan image showing new onset edema with right-to-left shift.

drain management and further care. He had persistent elevations in intracranial pressure (ICP) despite mannitol, bilateral EVDs, and chemical paralysis. He was then induced into a coma via phenobarbital continuous infusion for three days. Antifungal coverage was later changed to voriconazole for improved CNS penetrance in addition to amphotericin B until the development of an acute kidney injury. Intraoperative tissue cultures were positive for a dematiaceous fungus which would later speciate into Bipolaris. After cultures were finalized, the patient was continued on voriconazole $300 \mathrm{mg}$ BID monotherapy which he received for the remainder of his hospitalization. This antifungal regimen was based on clinical data reviews in conjunction with our infectious disease consultants.

The patient continued to deteriorate over the next 4-6 weeks. During this period, it was discovered from the patient's friend that prior to the onset of headaches, he had been inhaling garden mushrooms intranasally with cocaine. Given his young age and after extensive discussions with the patient's family, aggressive, 


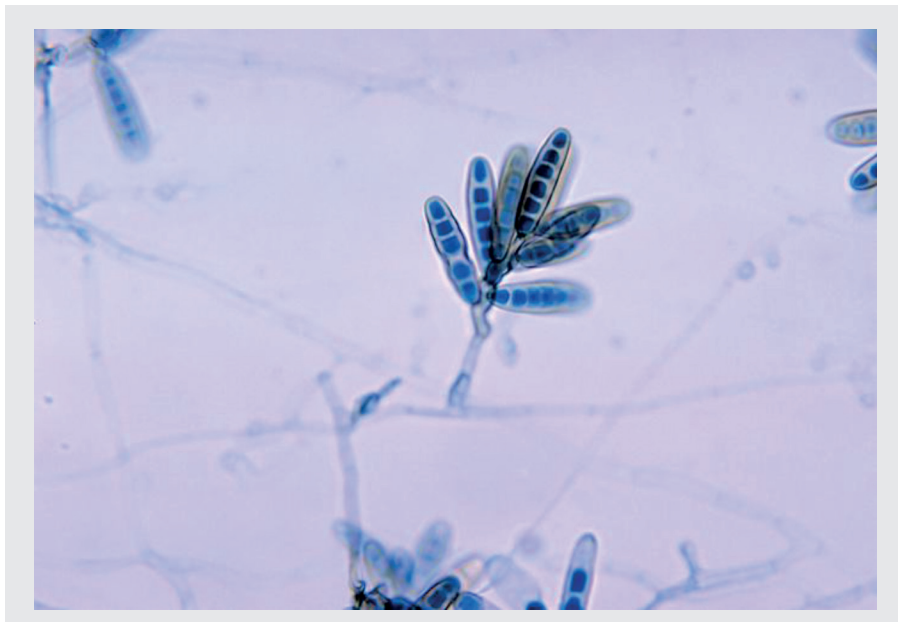

Figure 4. Bipolaris spp. ${ }^{11}$

life-sustaining measures were attempted to improve patient's clinical condition. He underwent bilateral ventriculo-peritoneal shunt placement, tracheostomy, and percutaneous endoscopic gastrostomy tube placement. The patient later developed Clostridium difficile infection, lung aspergillosis, and methicillinresistant Staphylococcus aureus pneumonia. After multiple conversations with the patient's family regarding worsening clinical status, he was transitioned to comfort measures under the supportive care service and died several days later.

\section{Discussion}

The dematiaceous fungi are pigmented fungi that represent a large and heterogeneous group of molds that cause a wide range of clinical pathologies. Among the most frequently isolated pathogens are Alternaria species, Bipolaris species, Curvulara species, Exophiala species, Madurella species, and Phialophora species. These organisms are present worldwide, found in soil, woods, and plants. ${ }^{1}$ Infection often results from traumatic implantation; our case represents CNS infection related to direct nasal inhalation. More commonly, these infections present with invasive sinusitis, allergic fungal sinusitis, ${ }^{2,3}$ or subclinical lung infection, possibly due to inhalation, since these fungal species are commonly found in household molds.
Interestingly, our case presented with isolated central nervous system infection without any evidence of sinus tract or respiratory disease. Most literature and previously documented case studies have demonstrated central nervous system infection in conjunction with disseminated disease from hematogenous spread. But in our case, the patient presented without evidence of systemic disease, as blood cultures for fungi remained negative throughout his admission.

Primary infection with dematiaceous fungi is typically seen in immunocompromised hosts, but primary CNS infection may be an exception to this rule. ${ }^{4}$ In a case review of 101 patients with cerebral phaeohyphomycosis, more than half had no underlying immunodeficiency or risk factor for infection. These primary CNS infections represent a significant challenge for physicians as case reports are few, and there are no established guidelines for anti-fungal therapy. In addition, there is no clear pathophysiology or mechanism leading to primary infection in the CNS. While one might assume local seeding from fungal sinusitis or otitis media, only one case report demonstrated a CNS infection with concomitant sinus infection, but in that case, the relationship was unclear as the patient had CNS infection on the opposite side of the sinus infection. ${ }^{5}$ This leads experts to believe that hematogenous spread is the most likely culprit, most commonly from a subclinical pulmonary infection. In our case, the patient had CT scans of his chest, abdomen, and pelvis upon arrival to our facility, which were negative for an infectious process. Our case is unique in that it demonstrated primary CNS infection by a mechanism which has not been previously described in literature.

A literature review of cerebral phaeohyphomycosis case reports and treatment strategies shows the prognosis of phaeohyphomycosis remains poor, with most outcomes resulting in death. ${ }^{6}$ One literature review showed that in 21 patients overall mortality was over $50 \%{ }^{7}$ with another study reported mortality as high as $79 \%,{ }^{4}$ regardless of the patients' being immunocompetent or immunocompromised. ${ }^{8}$ To reduce mortality, several therapeutic approaches have been attempted, but overall mortality remains high. Treatments have used amphotericin $\mathrm{B}$ alone or in combination with other azole medications. Voriconazole has shown some improved outcomes, likely due to its CNS penetrance 
and good oral bioavailability. However, more studies are needed to determine overall success. One study did report some survival benefits from a treatment of amphotericin B, 5-flucysteine, and itraconazole, but that study did not include CNS infection and focused primarily on soft tissue phaeohyphomycosis. ${ }^{9}$

\section{Conclusion}

In this case report, we describe a healthy young man who presented for evaluation of nausea, vomiting, and headache with a CT head scan demonstrating a massive right frontal mass with an unclear etiology. This mass turned out to be a fungal abscess leading to a diagnosis of cerebral phaeohyphomycosis. Despite aggressive surgical and non-surgical management, the patient unfortunately succumbed to his infection. This case report highlights a rare condition with an uncommon mechanism of inoculation via nasal inhalation and may prompt early recognition in future cases. We hope that as more cases are reported and reviewed we can establish clear treatment and antimicrobial regimens to reduce the overall mortality of this deadly infection.

Article citation: Daniele C, Ferguson M, Phillips C, Lachmansingh J, Nichols J, Fisher J, Holstead B, Opoku A. Novel origin of cerebral phaeohyphomycosis: a case report. The Southwest Respiratory and Critical Care Chronicles 2020;8(33):52-55

From: Departments of Internal Medicine (JN, JF, BH, AO) and Anesthesiology (CD, MF, CP, JL), Texas Tech, etc.

Submitted: $12 / 8 / 2019$

Accepted: $1 / 15 / 2020$

Reviewer: Kenneth Nugent MD

Conflicts of interest: none

This work is licensed under a Creative Commons Attribution-ShareAlike 4.0 International License.

\section{REFERENCES}

1. Brandt ME, Warnock DW. Epidemiology, clinical manifestations, and therapy of infections caused by dematiaceous fungi. J Chemother 2003 Nov;15 Suppl 2:36-47.

2. deShazo RD, Chapin K, Swain RE. Fungal sinusitis. N Engl J Med 1997 Jul 24;337(4):254-9.

3. deShazo RD, O'Brien M, Chapin K, et al. A new classification and diagnostic criteria for invasive fungal sinusitis. Arch Otolaryngol Head Neck Surg 1997 Nov;123(11):1181-8.

4. Revankar SG, Sutton DA, Rinaldi MG. Primary central nervous system phaeohyphomycosis: a review of 101 cases. Clin Infect Dis 2004,38(2):206-216.

5. Brown JW III, Nadell J, Sanders CV, et al. Brain abscess caused by Cladosporium trichoides (bantianum): a case with paranasal sinus involvement. South Med J 1976;69:1519-21.

6. Hironaga M, Watanabe S. Cerebral phaeohyphomycosis caused by Cladosporium bantianum: a case in a female who had cutaneous alternariosis in her childhood. Sabouraudia 1980 Sep;18(3):229-35.

7. Filizzola M, Martinez F, Rauf S. Phaeohyphomycosis of the central nervous system in immunocompetent hosts: Report of a case and review of the literature. Inter J Infect Dis 2004; 7:282-6.

8. Revankar S, Patterson JE, Sutton DA, et al. M. Disseminated phaeohyphomycosis: review of an emerging mycosis. Clin Infect Dis 2002;34:467-476.

9. Sharkey PK, Graybill JR, Rinaldi MG, et al. Itraconazole treatment of phaeohyphomycosis. J Am Acad Dermatol 1990 Sep;23(3 Pt 2):577-86.

10. Espinel-Ingroff A. Comparison of in vitro activities of the new triazole SCH56592 and the echinocandins MK-0991 (L-743,872) and LY303366 against opportunistic filamentous and dimorphic fungi and yeasts. J Clin Micro 1998.

11. Public Health Image Library (PHIL). Centers for Disease Control and Prevention, Centers for Disease Control and Prevention, 1971, phil.cdc.gov/Details.aspx?pid=4338. 